\title{
The Trisection of an Arbitrary Angle: A Classical Geometric Solution
}

\author{
Arthur Clair Rediske \\ General Electric Company: Retired \\ Olympia, Washington, USA \\ art1rediske@gmail.com
}

\begin{abstract}
This paper presents an elegant classical geometric solution for solving the ancient Greek's problem of angle trisection. Its primary objective is to provide a provable construction for resolving the trisection of an arbitrary angle, based on the restrictions governing the problem [1]. The angle trisection problem is believed to be unsolvable for compass-straightedge construction. As stated by Pierre Laurent Wantzel, (1837), the solution of the angle trisection problem corresponds to an implicit solution of the cubic equation; $x^{3}-3 x-1=0$, which is algebraically irreducible, and so is the geometric solution of the angle trisection problem [2]. The goal of the presented solution is to show the possibility to solve the trisection of an arbitrary angle using the traditional Greek's tools of geometry (a classical compass and straightedge). This paper changes the problem from the algebraic impossibility classification to a solvable plane geometrical problem. Fundamentally, this novel work is based on the fact that, algebraic irrationality is not a geometrical impossibility, as demonstrated in [3]. The exposed methods of proof have been reduced to the Euclidean postulates of classical geometry.
\end{abstract}

Keywords: Angle trisection; Geometric impossibility; An arbitrary angle; A angle; Compass; Straightedge; Classical geometry; Irrational numbers

SUBJECT CLASSIFICATION: Geometric solution of classical Greek problem of angle trisection.

Supporting Agencies: None

Language: English

Date of Publication: 2018-04-30

DOI: $10.24297 / j a m . v 14 i 2.7402$

ISSN: 2347-1921

Volume: 14 Issue: 02

Journal: Journal of Advances in Mathematics

Website: https://cirworld.com

This work is licensed under a Creative Commons Attribution 4.0 International License. 


\section{INTRODUCTION}

Angle trisection is one of the three classic problem of compass and straightedge constructions of ancient Greek mathematics [1]. It concerns construction of an angle equal to one third of a given arbitrary angle, using only two tools: an unmarked straightedge, and a compass. The problem is stated to be generally impossible to solve, as shown by (Pierre Laurent Wantzel, 1837), [2]. Wantzel's proof relies on ideas from the field of Galois theory - in particular to show that, trisection of an angle corresponds to the solution of a certain cubic equation ( $x^{3}-3 x-1=0$ ), which is not solvable using the given tools. The generic definition of the angle trisection problem is based on the assumption that: the trisection was to be exact according to plane geometry rules [3] and [4]. The rules stated that a point had no dimension, a line had no width. When a line intersected another line, a point was created. A compass could be used to measure a distance and then be used to create another distance equal to the first distance. A compass could be used to draw an arc or a circle. The straight edge could have no marks or indents. A quantum analysis of the angle trisection proof of impossibility expose that the proof pose a serious geometrical misconception. The proof does not take into account, the above stated governing conditions in the sense that, it is purely algebraic. It assumes that, for one to trisect an angle, the size of the angle has to be known. This is a very wrong perception. Euclidean geometry is typically classical. No measurement and arithmetic is allowed. It is based on logical reasoning, and the governing proofs are purely geometrical. Through the year 2017, several researchers; [5], and [6], have published a high profile scientific refutes against the angle trisection impossibility statement. [9] and [14] provides a clear description of Euclidean geometry, interpretation of the governing rules, and the differences between Euclidean geometry and Solid geometry, together with the other types of geometry, wellconstructed. A clear account of Euclidean and non-Euclidean geometry is found in the treatise; the Euclid's elements, translated and interpreted by different mathematics generations into various fashions as in [11]. This paper corresponds to this consideration, in presenting an alternative classical geometric solution for the stated old age problem of Greeks' mathematics. It is equally considered that, as demonstrated in [1], [2], [13], the angle trisection impossibility proof is not geometrical, but, algebraic, an approach completely prohibited in classical geometric constructions. The provided proofs is virtuously classical based on proportion of some quantities such as angles and triangles. Those commensurate imply quantities of similar magnitude, otherwise, no equality. This approach is in harmony with the consideration that, all classical geometrical problems should be sought geometrically [10]. In his Book III of La Géométrie [10], Descartes gave an account on the roots of cubic and quartic equations. He considers polynomials with integer coefficients. It is stated that, if there is an integer root, that gives a numerical solution to the problem, but, if there are no integral roots, the solutions must be constructed geometrically. Sections 1.1 and 1.2 discuss the geometrical foundations fundamentally followed in this work, and characterization of the methodology respectively.

\section{MATERIALS AND METHODS}

This section presents the proposed methodology. The presented contents depend upon the discussion in sections 1.1 through 1.5. It is reflected that, if an arc were drawn, using the apex of an unknown angle opening, to intersect the two rays forming the angle, the arc could be divided into four equal parts; then one part of the four could be subtracted, leaving three congruent parts. If the three congruent parts could be moved closer to an exact position relative to the apex of the angle, three congruent triangles could be created and therefore, the original unknown angle would be divided into three equal parts.

\subsection{Geometry Foundations}

The provided constructions are theoretically established based on the following geometrical facts:

1. Mirror Image-each side of a fiducial or reflector line is the reflection of the other side.

2. Corresponding line segments and angles of the mirror image are congruent.

3. Reflexive property - any segment or angle is congruent to itself.

4. A quadrilateral has four straight sides.

5. A parallelogram is a quadrilateral that has two pairs of parallel sides. 
6. One pair of opposite sides is parallel and equal in length.

7. If a traverse intersects two parallel lines, alternate interior angles are congruent.

\section{$1.2 \quad$ Notations}
1. $\angle \quad$ Angle notation
2. $\overline{A B} \quad$ Used to denote a straight line segment and a length
3. $\widehat{D E}$ and $\widehat{a-a}$ Used to denote a curve
4. $\cong$ Congruent
5. $\triangle$ Used to denote a triangle
6. $\quad \| \quad$ Used to denote parallelism

\subsection{Characterizations}

The elementary proof of the proposed methodology is based on some geometrical quantities such as triangles and other quadrilaterals characterized by the following properties. This depicts the classical geometric validity of the methods, in accordance to Euclidean ways of proofing geometrical analogies. It is considered that, a simple (non-intersecting) quadrilateral is a parallelogram if and only if any one of the following statements is true:

1. Two pairs of opposite sides are equal in length.

2. Two pairs of opposite angles are equal in measure.

3. The diagonals bisect each other.

4. One pair of opposite sides is parallel and equal in length.

5. Adjacent angles are supplementary.

6. Each diagonal divides the quadrilateral into two congruent triangles.

7. The sum of the squares of the sides equals the sum of the squares of the diagonals. (This is the parallelogram law.)

\subsection{Important Note on Rectangle Properties}

Among the elemental objects to be produced in the construction is a rectangle. For justification, a rectangle must be a parallelogram having the following properties, which will help in the results verification:

1. A pair of sides both parallel and congruent.

2. Contains at least one right angle.

3. Diagonals are congruent and bisect each other.

\subsection{Hypothesis}

The logic behind the proposed angle trisection solution has the foundation based on: "I was listening to a course on calculus and one statement stuck with me. The teacher said, 'Don't forget to subtract the starting point.' That statement provided the key to complete my solution of how to trisect an unknown angle using only a straight edge, a compass, and following all the rules of geometry." The solution takes an angle of unknown degrees; but no greater than 90 degrees, splits it into four congruent angles inside the original angle, then removes one of the smaller angles, leaving the remaining three congruent angles. Next, the three congruent angles have three congruent bases attached to them to form three congruent isosceles triangles, as illustrated from section 3.0. Then, as the base of each triangle, whose lengths do not change, is moved closer to the apex of the main angle, this shortens each side of all three triangles and widens the apex angle of each. There is only one place, where the following properties are met:

1. The endpoints of the bases of these three triangles touch each other from left to right.

2. The left-most and right-most bases intercept the two sides of the original unknown angle. 
3. Four lines form the sides of the three congruent isosceles triangles.

4. The apexes of these three triangles all touch the apex of the original unknown angle.

5. The outer lines of the left- and right-most triangles are the same as the lines of the original unknown angle.

In other words, this repositioning results in an increase in the apex angle of each congruent isosceles triangle. It can now be proven that these three isosceles triangles are congruent-i.e. they are identical in size-thereby proving the mathematical puzzle of trisecting an angle following the rules of the puzzle.

\section{RESULTS AND DISCUSSION}

\section{A. Results}

\subsection{Author's "Solution" to Trisection of an Angle}

The solution overview is as discussed in section 2.0. However, for angles over 90 degrees, one has to divide them in half and then trisect one of the halves. Two of these added together will be equal to one of the angles in the original larger angle.

\subsubsection{Trisection of Angles Less than $90^{\circ}$ (An Application of Art's "Solution")}

The solution is examined in the trisection of an angle less than $90^{\circ}$. Consider the following construction steps and the generated results:

1. Construct an angle with unknown, less than $90^{\circ}$ of opening (see Figure 1).

2. Label it $X A B C$. (Note: $X A$ will be used later.)

3. Open compass to approximately a 6 " opening.

4. Place sharp point of compass on Point $B$.

5. Swing compass to draw arc to intercept $\overline{A B}$ at Point $D$ and intercept $\overline{B C}$ at Point $E$. Bisect arc $\widehat{D E}$ :

6. Using Point $D$ and compass opening greater than $1 / 2 \widehat{D E}$, draw $\widehat{a-a}$.

7. With no change in compass opening, using Point $\mathrm{E}$, draw $\operatorname{arc} b-b$. Summary: An unknown angle now has an arc that intercepts the two legs.

8. Label the intersection points of the two arcs as Point 30.

9. Draw line from $B$, intersection of $\widehat{D E}$ at Point $F$, Point 30 , and terminate at Point 30 on $F F-F$.

10. Draw lines from Points $D$ and $E$ to Point 30.

11. Therefore, $\overline{30 D} \cong \overline{30 E}$ and $\overline{B D} \cong \overline{B E}$.

12. $\triangle D B 30 \cong \triangle E B 30$.

13. $\angle D B 30 \cong \angle E B 30$.

14. Therefore, $\overline{B-30}$ bisects angle $A B C$.

15. Therefore, $\widehat{D F} \cong \widehat{E F}$.

16. Draw chords $D F$ and $E F$.

17. Therefore, chords $D F$ and $E F$ are $\cong$.

NOTE: The above steps numbered 9 to 17 will be the guide to the reasons used to bisect the following six arcs: $\widehat{E F}, \widehat{D F}, \widehat{F H}, \widehat{G F}, \widehat{E H}$ and $\widehat{D G}$.

\section{Bisect $\widehat{E F}$.}

18. Using Point $E$ and compass opening greater than $1 / 2 \widehat{E F}$, draw $\widehat{c-c}$.

19. With no change in compass opening, using Point $F$, draw $\overline{d-d}$.

20. Label the intersection point of the two arcs as Point 31. 
21. Draw a line from Point $B$, intersection of $\widehat{D E}$ at Point $H$ and Point 31 .

22. $\overline{31 F} \cong \overline{31 E}$.

23. $\overline{B F} \cong \overline{B E}$.

24. $\triangle 31 B F \cong \triangle 31 B E$.

25. $\angle \mathrm{FB} 31 \cong \angle \mathrm{EB} 31$

26. Therefore, line $31 B$ bisects the $\angle F B E$.

27. Thus, $\widehat{F H} \cong \widehat{E H}$.

28. Draw chords $\overline{F H}$ and $\overline{E H}$.

29. Chords $\overline{F H}$ and $\overline{E H}$ are $\cong$. Bisect $\widehat{D F}$.

30. Using Point $F$ and compass opening greater than $1 / 2 \widehat{D F}$, draw $\widehat{e-e}$.

31. With no change in compass opening, using Point $D$, draw $\overline{f-f}$.

32. Label the intersection point of the two arcs as Point 32.

33. Draw line from Point $B$, intersecting $\widehat{D E}$ at Point $G$; continue to intersection of $\operatorname{arc} e-e$ and $\operatorname{arc} f-f$, which is Point 32.

34. $\overline{32 F} \cong \overline{32 D}$.

35. Line $B F \cong$ Line $B D$.

36. $\triangle 32 F B \cong \triangle 32 D B$.

37. $\angle F B 32 \cong \angle D B 32$.

38. $\overline{32 B}$ bisects the $\angle D B F$.

39. Therefore, $\widehat{D G} \cong \widehat{F G}$.

40. Draw chords $\overline{D G}$ and $\overline{F G}$.

41. Chords $\overline{D G}$ and $\overline{F G}$ are $\cong$.

Bisect $\widehat{\boldsymbol{E H}}$ (see Figure 2). (Note: chord HS and chord ES to be used later in proof.)

42. Using Point $H$ and compass opening to greater than $1 / 2$ chord $E H$, draw $\overline{k-k}$.

43. Using Point $E$, no change on compass opening, draw $\overline{l-l}$.

44. Label intersect point of two arcs as Point 35.

45. Draw line from $B$, to Point 35 , intersecting chord $\mathrm{EH}$ at Point $S$.

46. Line $35 \mathrm{E} \cong$ Line $35 \mathrm{H}$.

47. Line $B H \cong$ Line $B E$.

48. $\triangle H B 35 \cong \triangle E B 35$.

49. Line $B 35$ bisects $\angle H B E$.

50. Therefore, arc HS $\cong$ to arc ES.

51. Draw chords $\overline{H S}$ and $\overline{E S}$.

52. Chords $\overline{H S}$ and $\overline{E S}$ are $\cong$.

Bisect $\widehat{\boldsymbol{D G}}$. (Note: $\overline{D T}$ and $\overline{G T}$ to be used later in proof.)

53. Using Point $G$ and compass opening to greater than $1 / 2 \operatorname{arc} \mathrm{DG}$, draw $\overline{n-n}$.

54. Using Point $D$, no change in compass opening, draw $\widehat{\overline{m-m}}$.

55. Label the intersection of the two arcs as Point 36.

56. Draw line from Point $B$ to Point 36, intersecting arc DG at Point $T$.

57. Line $36 D \cong$ Line $36 G$. 
58. $\triangle G B 36 \cong \triangle D B 36$.

59. Line $B 36$ bisects the $\angle D B G$.

60. Draw chords $\overline{D T}$ and $\overline{G T}$.

61. Chords $\overline{D T}$ and $\overline{G T}$ are $\cong$.

\section{Bisect $\widehat{F H}$ :}

62. Using Point $F$ and compass opening to greater than $1 / 2$ chord $\mathrm{FH}$, draw $\sqrt{h-h}$.

63. Using Point $H$, with no change in compass opening, draw $\widehat{g-g}$.

64. Label the intersecting point of the two arcs as Point 33.

65. Draw line from Point $B$, intersecting Line $F H$ at Point $J$ and Point 33.

66. $\overline{33 F} \cong \overline{33 H}$.

67. $\triangle 33 F B \cong \triangle 33 H B$.

68. $\angle F B 33 \cong \angle H B 33$.

69. $\overline{33 B}$ bisects $\angle F B H$.

70. Therefore, arc FJ $\cong$ arc HJ.

71. Draw chords $\overline{F J}$ and $\overline{H J}$.

72. Chords $\overline{F J}$ and $\overline{H J}$ are $\cong$.

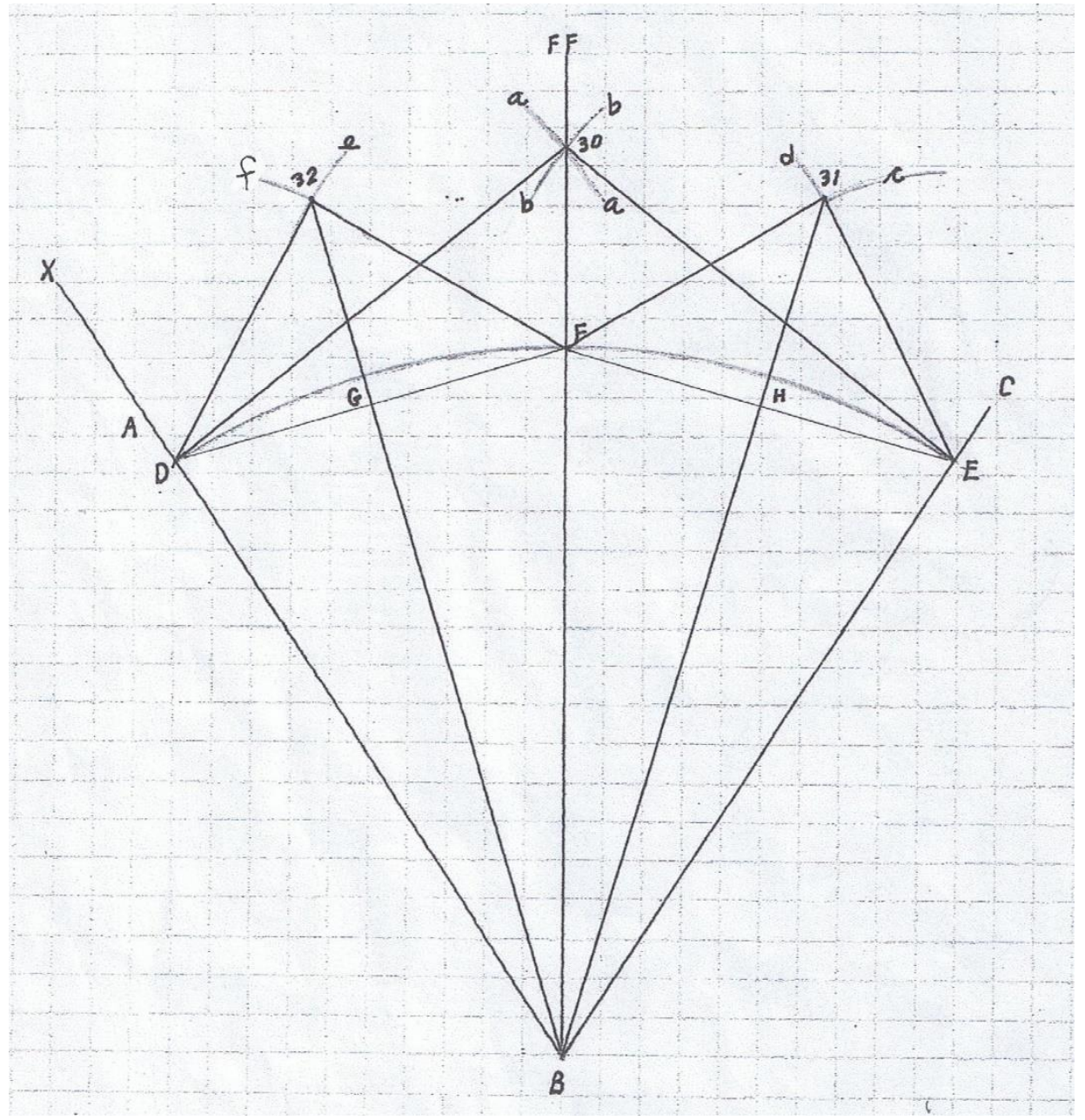

Figure 1: Two rays form an angle, arc added, angle divided into 4 congruent angles 


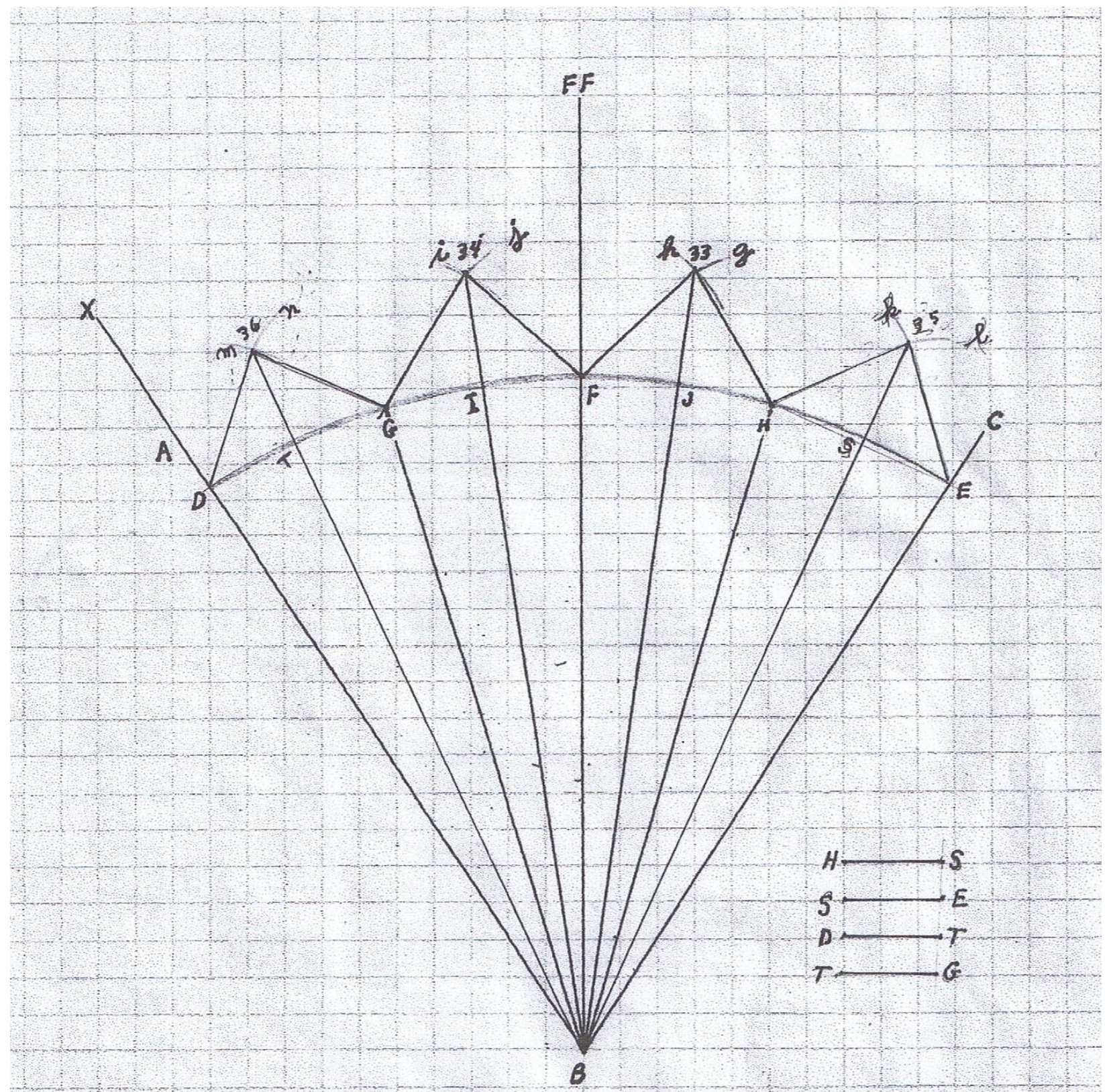

Figure 2: Four congruent angles divided into eight congruent angles. Four labeled segments used in illustrative proof 


\section{Bisect $\widehat{G F}$ :}

73. Using Point $G$ and compass opening to greater than $1 / 2 \operatorname{arc~FG}$, draw $\widehat{\imath-l}$.

74. Using Point $F$, with no change in compass, draw $\widehat{J-J}$.

75. Label the intersecting point of the two arcs as Point 34.

76. Draw line from Point $B$, intersecting arc FG at Point $I$ and Point 34 .

77. Therefore, $\overline{34 G} \cong \overline{34 F}$.

78. Therefore, $\overline{34 B}$ bisects $\angle G B F$.

79. Therefore, $\angle S D B G, G B F, F B H$, and $H B E$ are $\cong$

80. Therefore, $\angle G B I \cong \angle F B I$.

81. Therefore, $\angle F B J \cong \angle H B J$.

82. Draw auxiliary $\overline{D G W}$ to intercept $\overline{F-F F}$ at Point $W$ (see Figures 3 and 4).

83. Therefore, $\overline{D G}$ is the chord of $\widehat{D G}$.

84. Draw auxiliary $\overline{E H W}$ to intercept $\overline{F-F F}$ at Point $W$.

85. Therefore, $\overline{E H}$ is the chord of $\widehat{E H}$.

86. $\overline{G F}(-) \overline{G I}=\overline{I F 1 a}, \overline{F 1 a H}(-) \overline{J H}=\overline{F 1 a J}$.

87. $\overline{I F}(+) \overline{F 1 a J}=\overline{I J}$.

88. $\overline{I J}$ is the chord of $\widehat{I J}$.

89. $\overline{D G} \cong \overline{I J} \cong \overline{H E}$.

Summary: Steps $18-86$ bisected the arc $\widehat{D E}$ to create eight chords. Not all the chords will be used in the final proof. Figure 1 does not show the chords.

If chords $\overline{D T}(+) \overline{T G}=\overline{D G}, \overline{G I}(+) \overline{I F}=\overline{G F}, \overline{F J}(+) \overline{H J}=\overline{F H}, \overline{H S}(+) \overline{S E}=\overline{H E}$, then chords $\overline{D G}, \overline{G F}, \overline{F H}$, and $\overline{H S}$ are each $1 / 4$ of $\operatorname{arc} D E$.

If chord $\overline{I F}$ in chord $\overline{G F}$ is added to chord $\overline{F J}$ in chord $\overline{F H}$ and chords $\overline{G I}$ and $\overline{J H}$ are ignored, a new chord $\overline{I J}$ will be created and will be congruent to chords $\overline{D G}$ and $\overline{H E}$ for a total of three chords.

NOTE: Chords $\overline{G I}$ and $\overline{H J}$ will not be used and will not be referred to in the rest of this summary.

Designate line $\overline{B-F F}$ as a fiducial and reflection line, necessary for the production of mirror images.

\section{Make $\overline{G M} \| \overline{B D}$ :}

90. Open compass to less than chord DG. (Refer to Figure 3.)

91. With sharp point on Point $\mathrm{D}$, draw $\overline{k-k}$ to intersect $\overline{B D}$ at Point 20 , chord $\mathrm{DG}$ at Point 1.

92. With no change in compass opening, sharp point of compass on Point $\mathrm{G}$, draw $\overline{l-l}$ to intersect $\overline{G W}$ at Point 2.

93. Opening compass to $1-20$, sharp point of compass on Point 1 , draw $\widehat{p-p}$.

94. With no change in compass opening, sharp point on Point 2, draw $\widehat{n-n}$ to intersect $\widehat{l-l}$ at Point $y$.

95. Draw $\overline{G Y M}$ to intersect Point $y$ and terminate at Point $M$ on $\overline{B E}$.

96. $\angle 1 D 20 \cong \angle 2 G Y$.

97. Therefore, $\overline{G M} \| \overline{B A}$.

Summary: Steps 90 through 97 created a line \|| to one leg of the angle. 


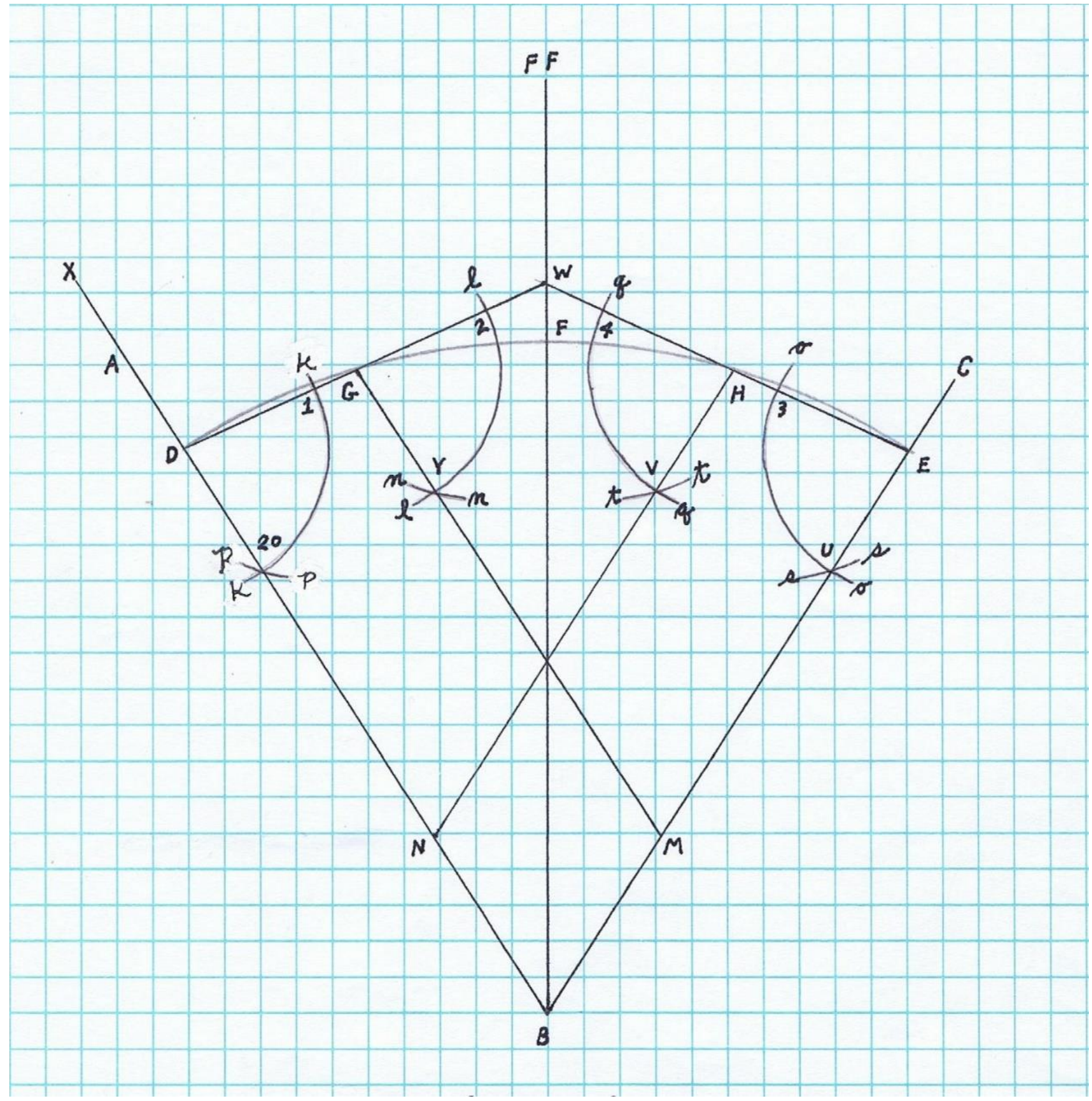

Figure 3: Constructing line G-M parallel to Line $D-B$, and line $H-N$ parallel to line $E-R$ 
Make $\overline{H N} \| \overline{E B}$ :

98. Opening compass to less than $\overline{E H}$, sharp point of compass on Point $E$, draw $\widehat{O-O}$ to intersect $\overline{E H}$ at Point 3 and $\overline{B E}$ at Point $U$.

99. With no change in compass opening, sharp point of compass on Point $H$, draw $\widehat{q-q}$ to intersect $\overline{H W}$ at Point 4.

100.100.Open compass width to $3-U$.

101. With no change in compass opening, sharp point of compass on Point 3 , draw $\widehat{s-s}$ to intersect Line $B E$ at Point $U$.

102. With no change in compass opening, sharp point of compass on Point 4 , draw $\overline{t-t}$ intersecting $\widehat{q-q}$ at Point $V$.

103.Draw $\overline{H V N}$ to intersect $\widetilde{q-q}$ at Point $V$, and terminate at Point $N$ on $\overline{B D}$.

104. $\angle E 3 U \cong \angle H 4 V$.

105. Therefore, $\overline{H N} \| \overline{B E}$.

Summary: Steps 98 through 105 created a line $\|$ to one leg of the angle.

Make $\overline{I K}$ and $\overline{J L} \|$ to $\overline{F F-B}$ (refer to Figure 4).

106. Draw auxiliary $\overline{J I S}$ to intersect $\overline{X D}$ at point $S$.

107. Line B-FF bisects arc $\widehat{I J}$ and chord $\overline{I J}$.

108. Open compass to less than $Z-I$, sharp point of compass on Point $Z$, draw $\overline{x-x}$ to intersect $\overline{Z I}$ at Point 6 and Line FF-Z at Point 5.

109. With no change in compass opening, sharp point of compass on Point $I$, draw $\overline{u-u}$ to intersect $\overline{I S}$ at Point 8.

110.With no change in compass opening, sharp point of compass on Point $J$, draw $\widehat{w-w}$ intersecting $\widehat{J-Z}$ at Point 10.

111.Open compass to Point 5 on $\overline{F F-Z}$ and Point 6 on $\overline{I-Z}$.

112. With no change in compass opening, sharp point of compass on Point 6 , draw $\overline{r-r}$ to intersect $\overline{F F-Z}$ at Point 5.

113. With sharp point of compass on Point 8 on $\overline{I-S}$, draw $\overline{Z-Z}$ to intersect $\overline{u-u}$ at Point 7 .

114. With sharp point of compass on Point 10 on $\overline{J-Z}$, draw $\overline{m-m}$ to intersect $\widehat{w-w}$ at Point 9.

115.Draw $\overline{7 I O K}$ that intersects $\overline{G M}$ at Point $O$.

116.Draw $\overline{9 J P L}$ that intersects $\overline{H N}$ at Point $P$.

117. Therefore, $\overline{I O K} \| \overline{F F Z B}$.

118. $\overline{J L} \| \overline{F F Z B}$.

119. Therefore, $\overline{I K} \| \overline{J L}$.

Summary: Steps 106 through 119 created two lines $\|$ to $\overline{B-F F}$ that will be used to create one pair of $\cong$ sides, in the first of three quadrilaterals.

120. Draw $\overline{O P}$.

121. $\overline{I J} \| \overline{O P}$ and $\overline{I O} \| \overline{J P}$.

122. Quadrilateral IOPJ is created.

Summary: Steps 120 through 122 created quadrilateral IOPJ.

\section{Prove IOPJ is a Parallelogram:}

123. Distance from $B$ to $I$ and $J \cong$.

124. Distance from $B$ to $O$ and $\mathrm{P} \cong$. 
125. $\overline{F F-B}$ is a fiducial and reflection line.

126. Therefore, $\overline{I O} \cong \overline{J P}$.

127. Therefore, IOPJ parallelogram is created.

128. Therefore $\overline{I J} \cong \overline{O P}$.

Summary: Steps 123 through 128, IOPJ verified as a parallelogram.

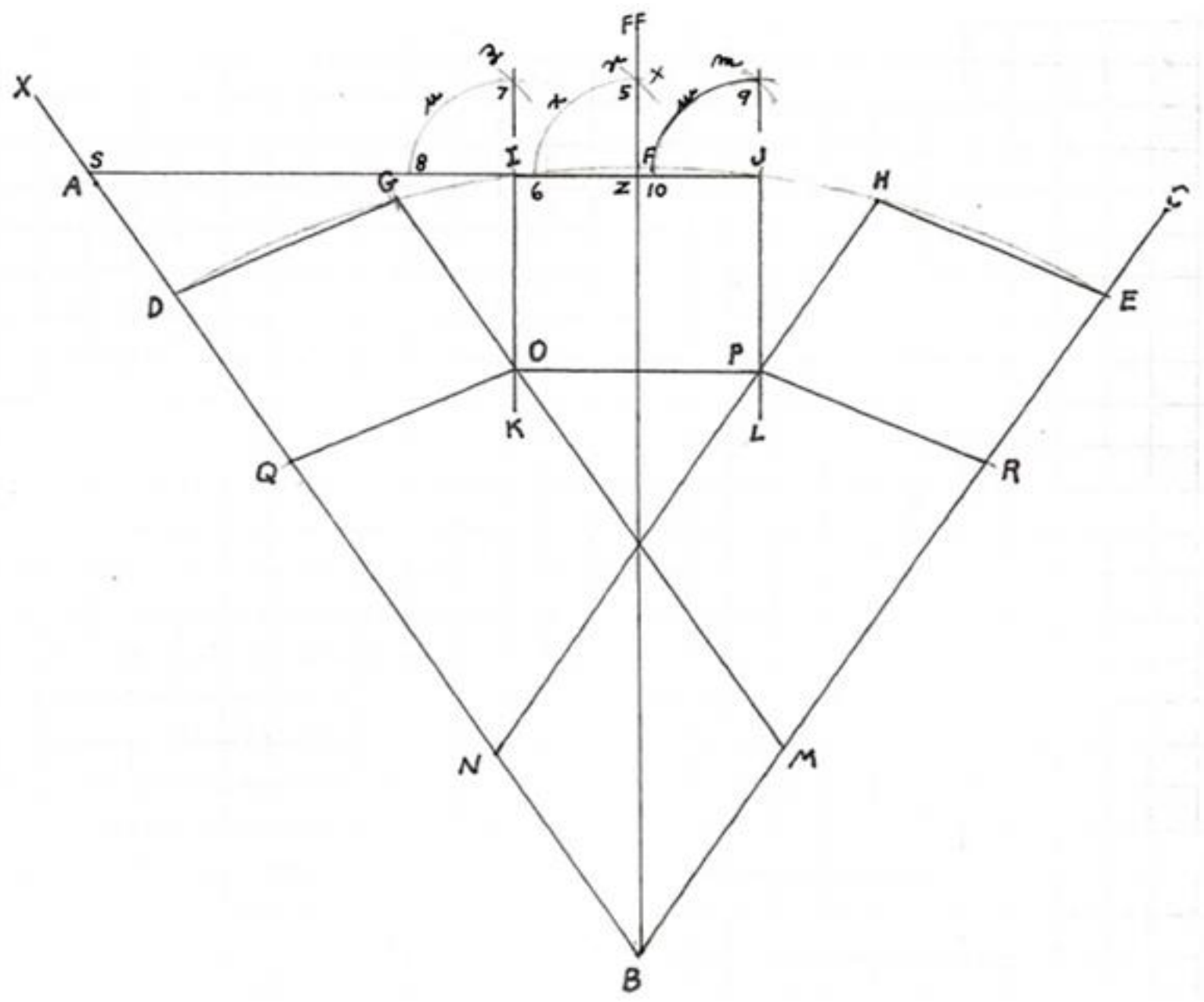

Figure 4: Constructing lines I-K and J-L parallel to fiducial mirror line FF-B.

Draw arc Q-O-P-R. H1-P-R-E1 not congruent to I-O-P-J

\section{Prove IOPJ is a rectangle:}

Note: The following steps numbered 126 through 137 will create eight right triangles, also used to construct a rectangle (see Figures 5 and 6 ).

129. Draw $\overline{F 4-I}$, it is a shared hypotenuse of created $\triangle s F 4-I-O-\# 1$ and $F 4-I-F 1 a-\# 2$.

130. Draw $\overline{F 4-J}$, it is a shared hypotenuse of created $\triangle s F 4-J-F 1 a-\# 3$ and $F 4-J-P-\# 4$.

131. Draw $\overline{F 1 a-O}$, it is a shared hypotenuse of created $\triangle s F 1 a-O-I-\# 6$ and $F 1 a-O-F 4-\# 5$.

132. Draw $\overline{F 1 a-P}$, it is a shared hypotenuse of created $\triangle s F 1 a-P-F 4-\# 8$ and $F 1 a-P-J-\# 7$. 
133.I $-O-F 4-F 1 a$ is a rectangle (see Figure 7).

134.F1a-F4 $P-J$ is a rectangle.

135. Add together two rectangles $I-O-F 4-F 1 a$ and $F 1 a-F 4-P-J$.

136. Rectangle $F 1 a-F 4-P-J(+)$ Rectangle I-O-F4-Fla $\cong$ Rectangle $I-O-P-J$.

Mirror line $\overline{F F-B}$ is also a perpendicular bisector of $\overline{I-J}$ and $\overline{O-P}$.

The above 8 triangles are all right angles.

Each pair of triangles uses the same line for its hypotenuse and the heights are $\cong$ between two parallel lines.

\section{Creation of Quadrilateral EHPR:}

137. Open compass to $\overline{B O}$.

138. With sharp point on Point $B$, establish Point $R$ on Line $B C$.

139. Draw $\overline{P R}$.

140.Quadrilateral EHPR is created.

Summary: Steps 137 through 140 created quadrilateral EHPR.

Because quadrilateral $E H P R$ is not a mirror image of rectangle IOPJ, EHPR must have a shape change (refer to Figure 5).

141.Open compass to approximately $3 / 4 \overline{P R}$.

142. With sharp point on Point $R$, draw $\widehat{t-t}$.

143. With sharp point on Point $P$, draw $\widehat{s-s}$ to intersect $\widehat{t-t}$ at Point $U$.

144.Draw $\overline{B-u-x-Z 1 a}$ to intersect $\widehat{D E}$ at Point $Z 1$.

145.Open compass to $\overline{H S}$ (refer to Figure 2).

146. Wish sharp point on Point Z1, transfer compass opening to Point E1 on $\widehat{D E}$.

147.Open compass to $\overline{E S}$ (refer to Figure 2).

148. With sharp point on Point $Z 1$, transfer compass opening to Point $H 1$ on $\widehat{D E}$.

149.Draw auxiliary lines $\overline{H 1-P}, \overline{E 1-R}, \overline{H 1-E 1}$.

Summary: Steps 141 through 149 created quadrilateral H1-P-R-E1.

\section{Constructing shared diagonals:}

150.Draw $\overline{X-H 1}$. It is a shared hypotenuse of created $\triangle s X-H 1-P-\# 9$ and $X-H 1-Z 1 a-\# 10$ (see Figure 8).

151.Draw $\overline{X-E 1}$. It is a shared hypotenuse of created $\triangle s X-E 1-Z 1 a-\# 11$ and $X-E 1-R-\# 12$.

152.Draw $\overline{Z 1 a-P}$. It is a shared hypotenuse of created $\triangle s Z 1 a-P-H 1-\# 13$ and $Z 1 a-P-X-\# 14$ (see Figure 9).

153. Draw $\overline{Z 1 a-R}$. It is a shared hypotenuse of created $\triangle s Z 1 a-R-X-\# 15$ and $Z 1 a-R-E 1-\# 16$.

154.H1 $-P-X-Z 1 a$ is a rectangle (see Figure 10).

$155 . Z 1 a-X-R-E 1$ is a rectangle. 


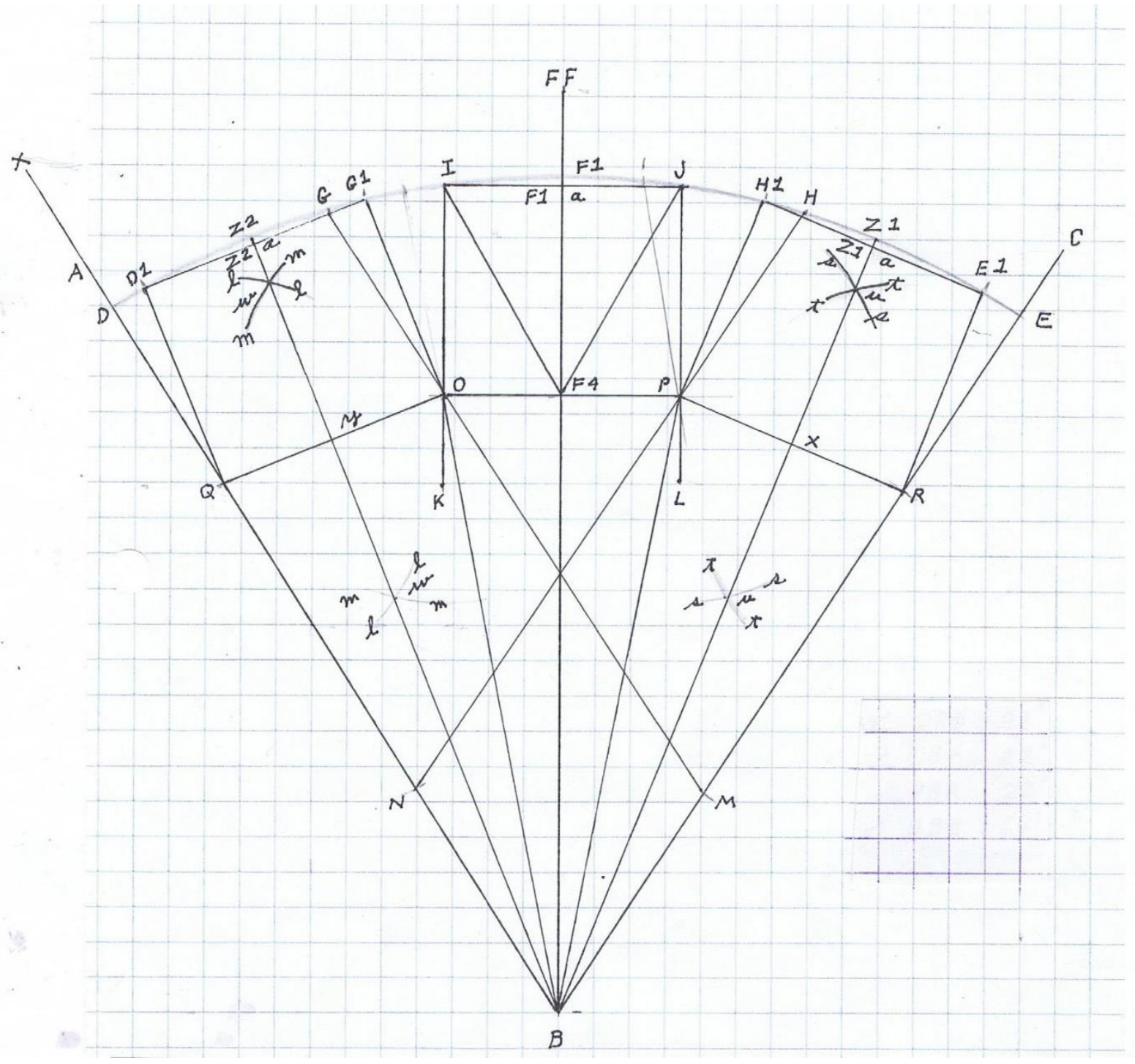

Figure 5: Constructing first diagonals I-F4 and J-F4. 


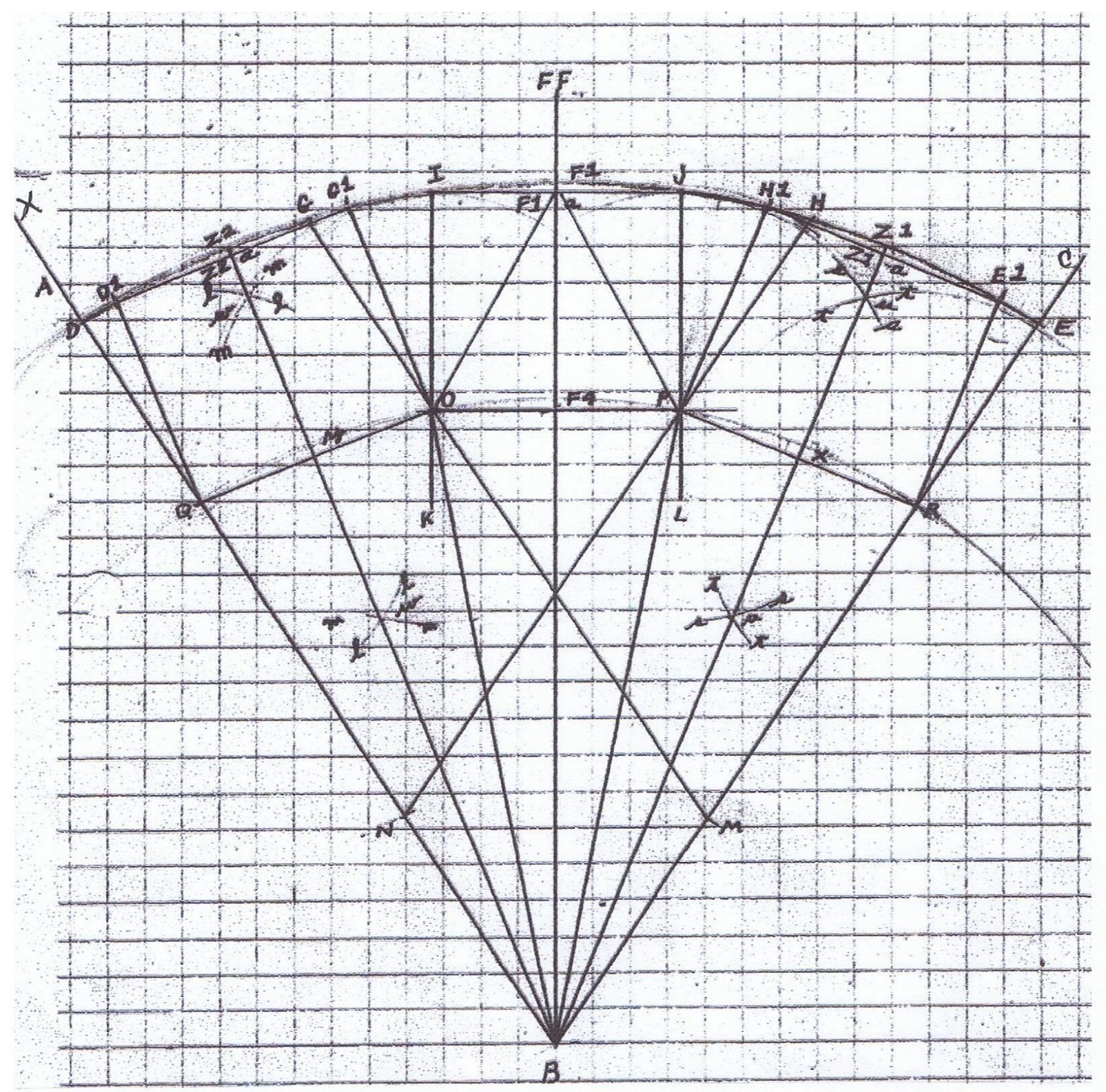

Figure 6: Constructing last two diagonals $\mathrm{O}-\mathrm{F} 1 \mathrm{a}$ and P-F1a. 


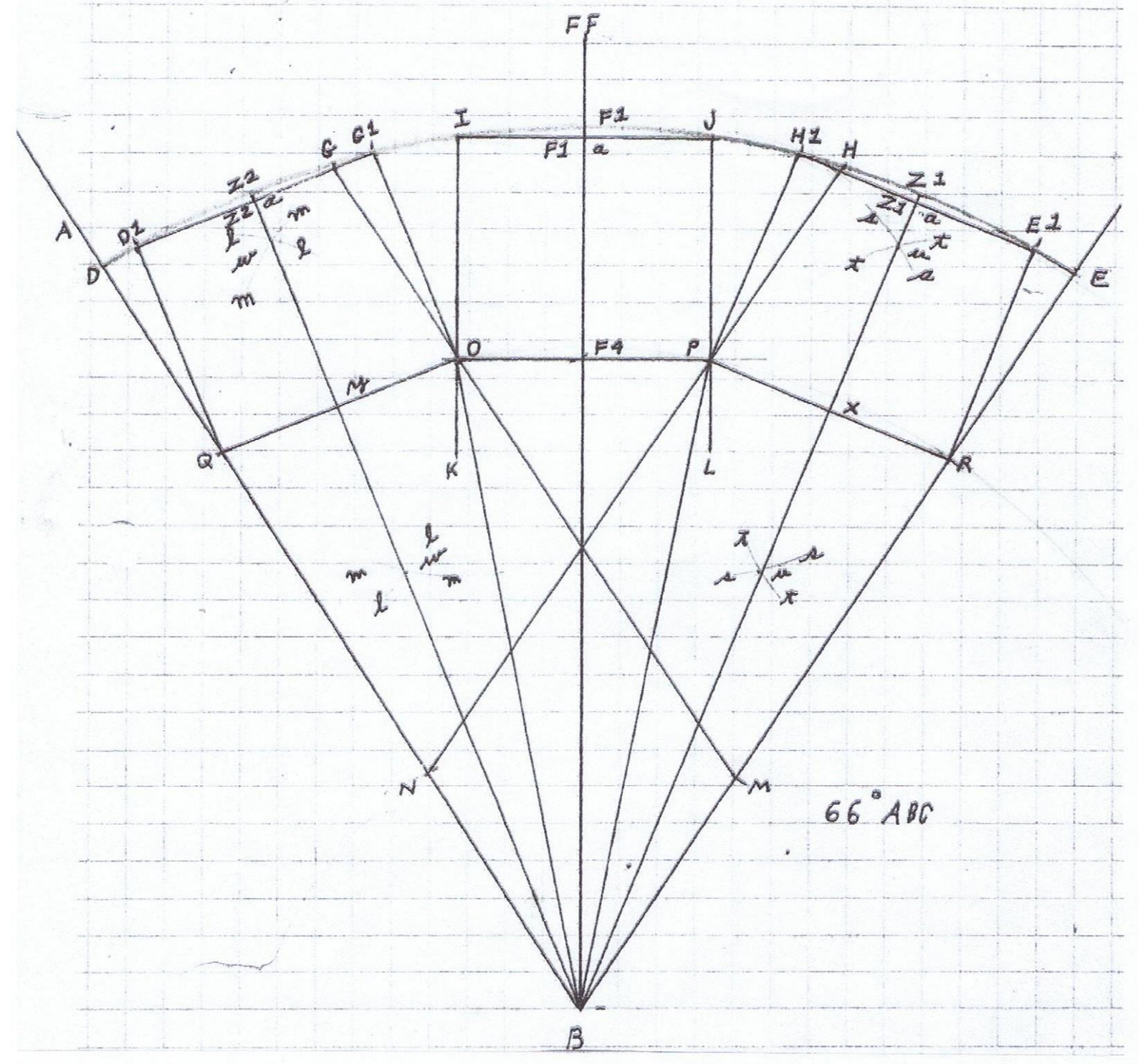

Figure 7: Determine bisecting lines of three angles, before shape changes.

Mirror line, $B-X-Z 1 a$ is also a perpendicular bisector of lines $\overline{H 1-E 1}$ and $\overline{P-R}$.

The above 8 triangles are all right triangles.

Each pair of triangles uses the same line for its hypotenuse and heights are $\cong$ between two parallel lines.

156. Combine rectangles $H 1-P-X-Z 1 a$ and $Z 1 a-X-R-E 1$.

157. Rectangle H1-P-R-E1 is created, and is $\cong$ to IOPJ.

158. Using $B-F F$ line as reflection, use similar steps as above and make quadrilateral $D Q O G$ into rectangle $D 1-Q-O-G 1$, mirror image of $H 1-P-R-E 1$.

Summary: There are now three $\cong$ rectangles.

159. Therefore, $D 1-Q-O-G 1, I-O-P-J, H 1-P-R-E 1$ are $\cong$. 
160. Therefore, cords D1-G1, Q-O, I-J, O-P, H1-E1, and P-R are $\cong$.

161. Therefore, Lines $B Q, B O, B P, B R$ are $\cong$.

162. Therefore, $\triangle S Q B O, O B P, P B R$ are $\cong$

163. Therefore, $\angle S Q B O, O B P, P B R$ are $\cong$. Therefore $\angle A B C$ is trisected into three congruent angles.

164. Therefore Angle $A B C$ is trisected into three congruent angles.

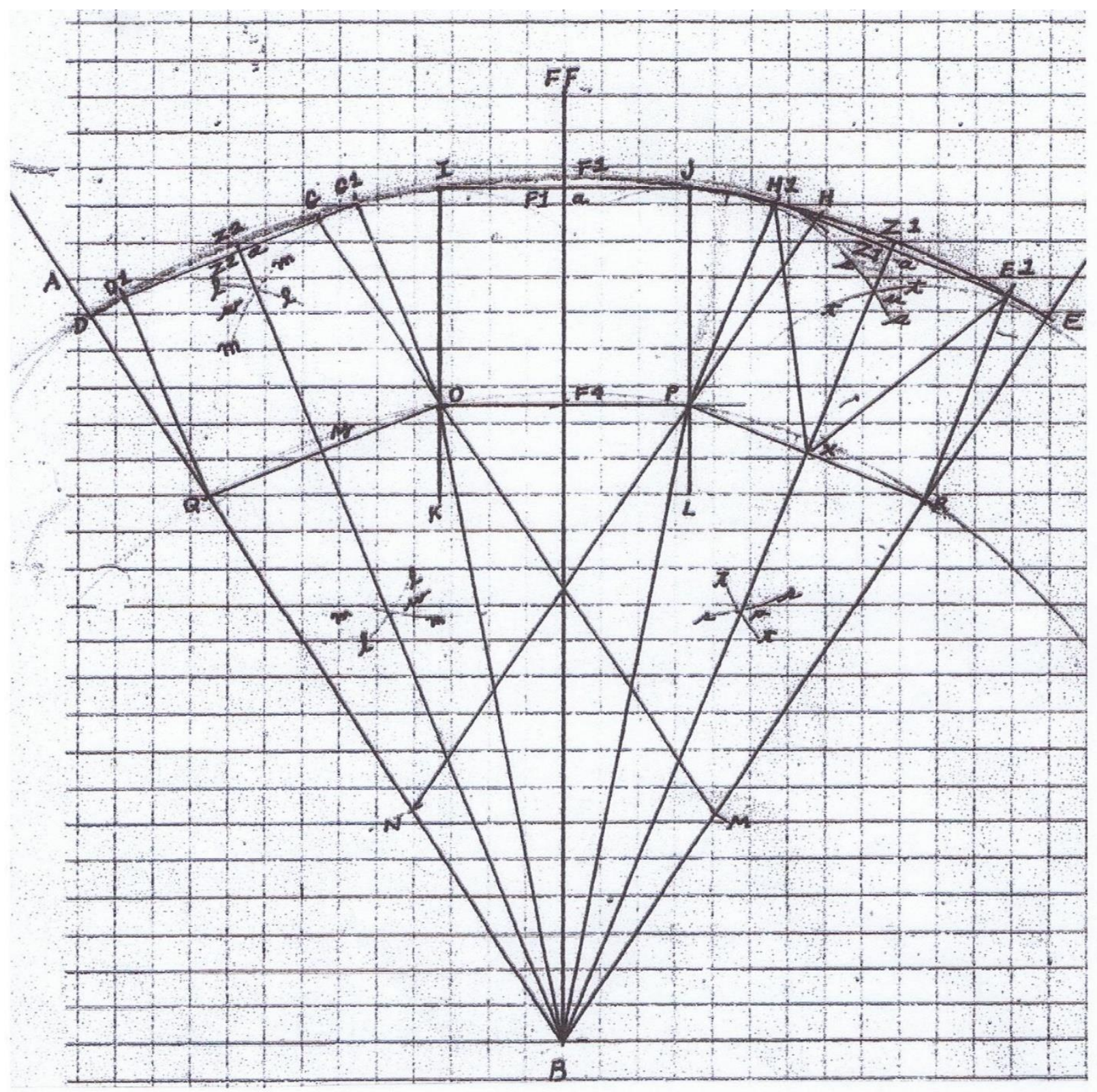

Figure 8: Constructing fist two diagonals $\mathrm{H} 1-\mathrm{X}$ and $\mathrm{E} 1-\mathrm{X}$. 


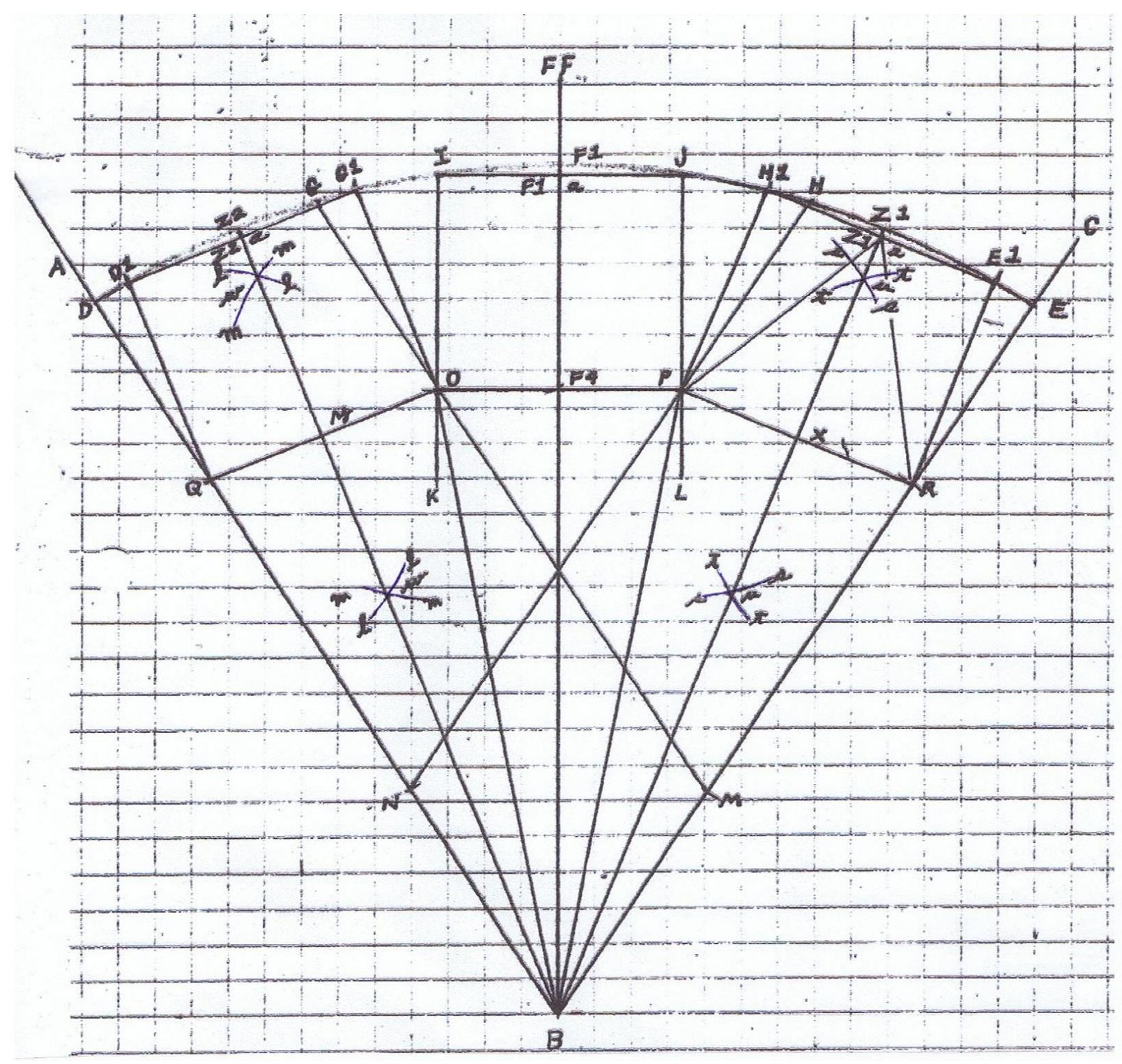

Figure 9: Constructing last two diagonals P-Z1a and R-Z1a. 


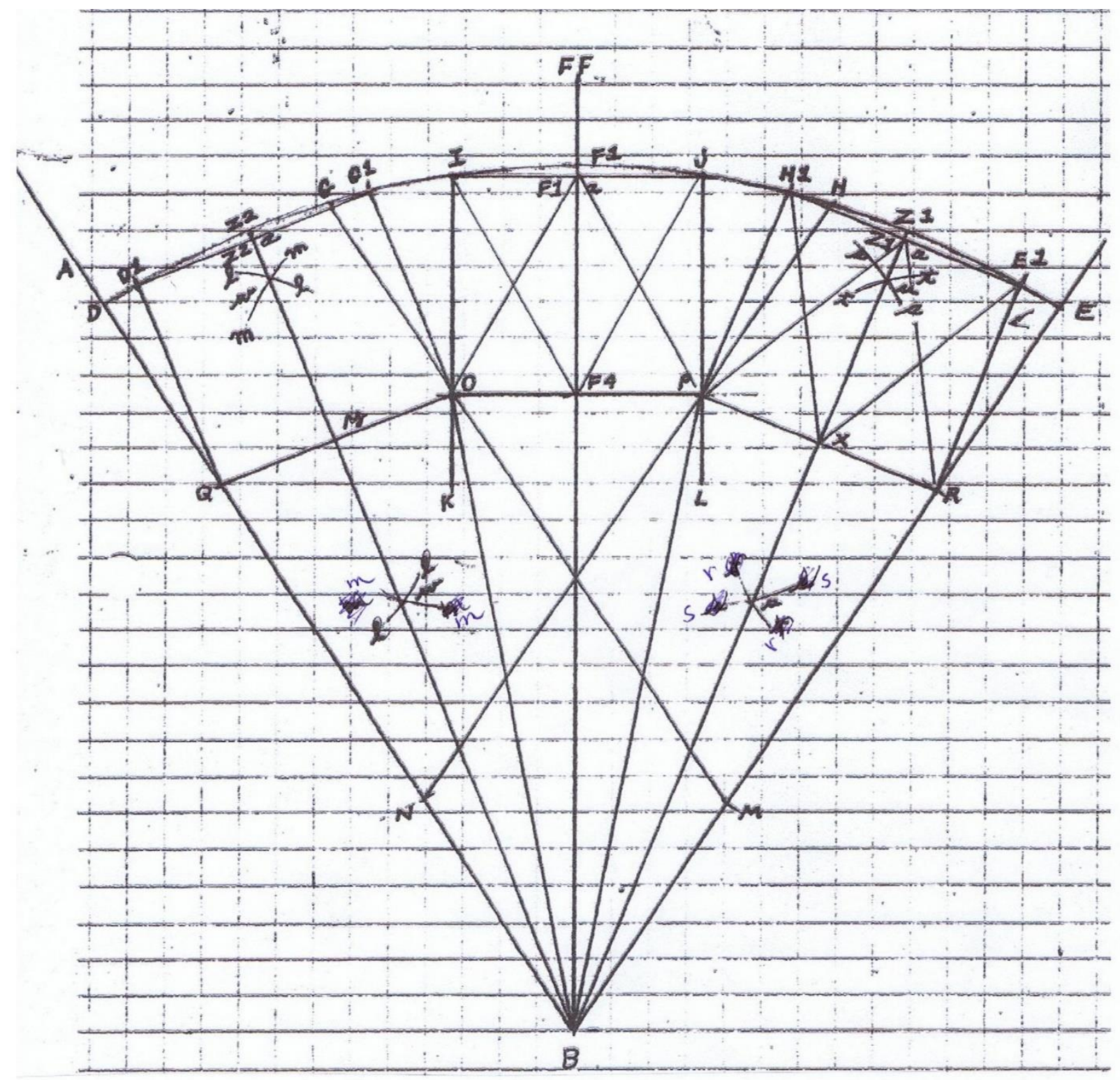

Figure 10: Four congruent rectangles.

\section{B. Discussion}

Throughout the presented methodology, it has been shown that the angle trisection problem is solvable using the proposed method of construction. The representation of results in this work is from figure (1) to figure (10). Figures (1) through (10) presents the obtained construction results, based on Euclidean rigor of classical geometry. All the work constructions are performed classically, using compass-straightedge techniques as set in [11]. Figure (1) presents the basic geometric construction, involving definition of quantities such as angles, and the characteristic properties. Figures (2), (3), and (4) exhibit the application of the generic Euclid postulates, implying the practical aspect of the method. The very significant consideration employed in this work is the logic that the problem of trisecting an arbitrary angle could be solved if a particular algorithm resolves the trisection of two known angles, without altering the construction principal. Figures (5), (6), and (7) create right triangles, which are then used to construct a rectangle. Figures (8), (9), and (10) create shared diagonals. Figures (11) through (13) show the geometric results of trisecting angles of $48^{\circ}$, $60^{\circ}$ and $72^{\circ}$ 


\section{《(⿶) $\rfloor$}

respectively, employing the author's method. Figure (14) shows the traditional Euclidean approach of the proof, based on the Pythagoras theorem, which exhibits use of proportions to inspect if two quantities are commensurate or not. It is justified that the trisection of $66^{\circ}$ angle is correct, and so is the trisection of any other angle. The classical geometric trisection of any angle greater than $90^{\circ}$ in magnitude, as stated earlier, could be performed by dividing the angle in half and then trisecting one of the halves using the proposed approach. Two of those angles added together will be equal to one of the angles in the original large angle.

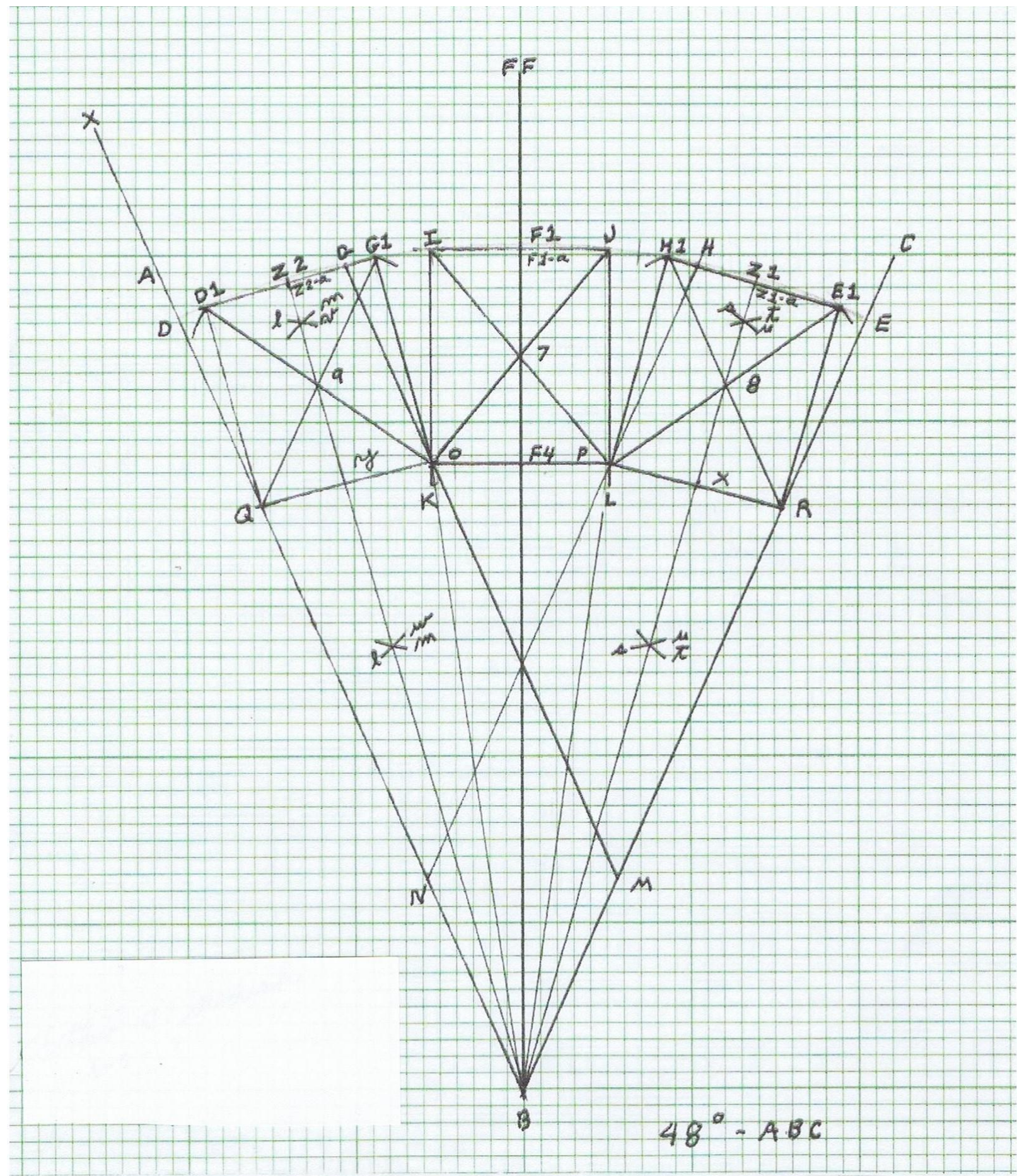

Figure 11: Solving the trisection of a 48 degree angle. 


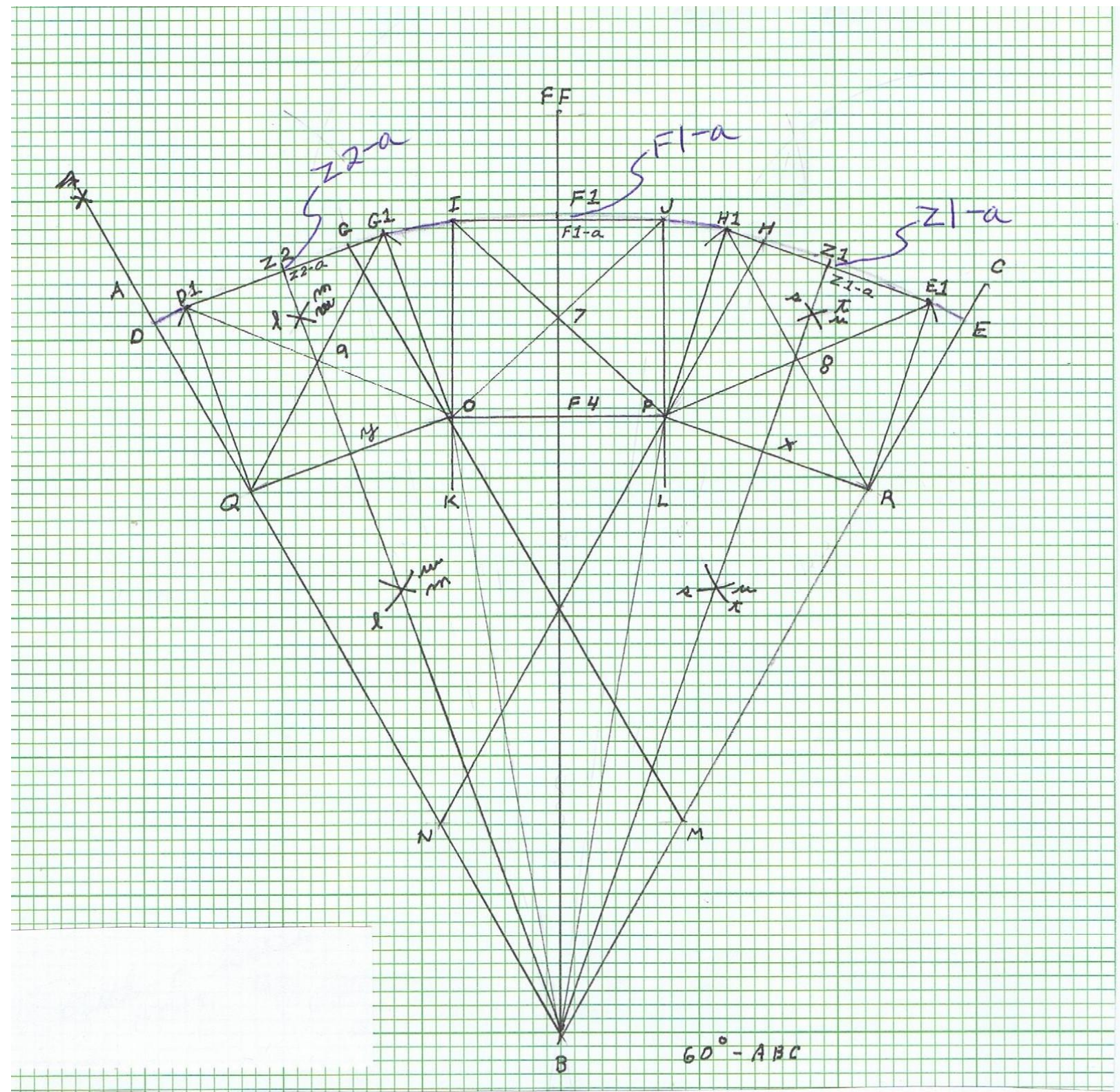

Figure 12: Solving the trisection of a 60 degree angle 


\section{《肉》}

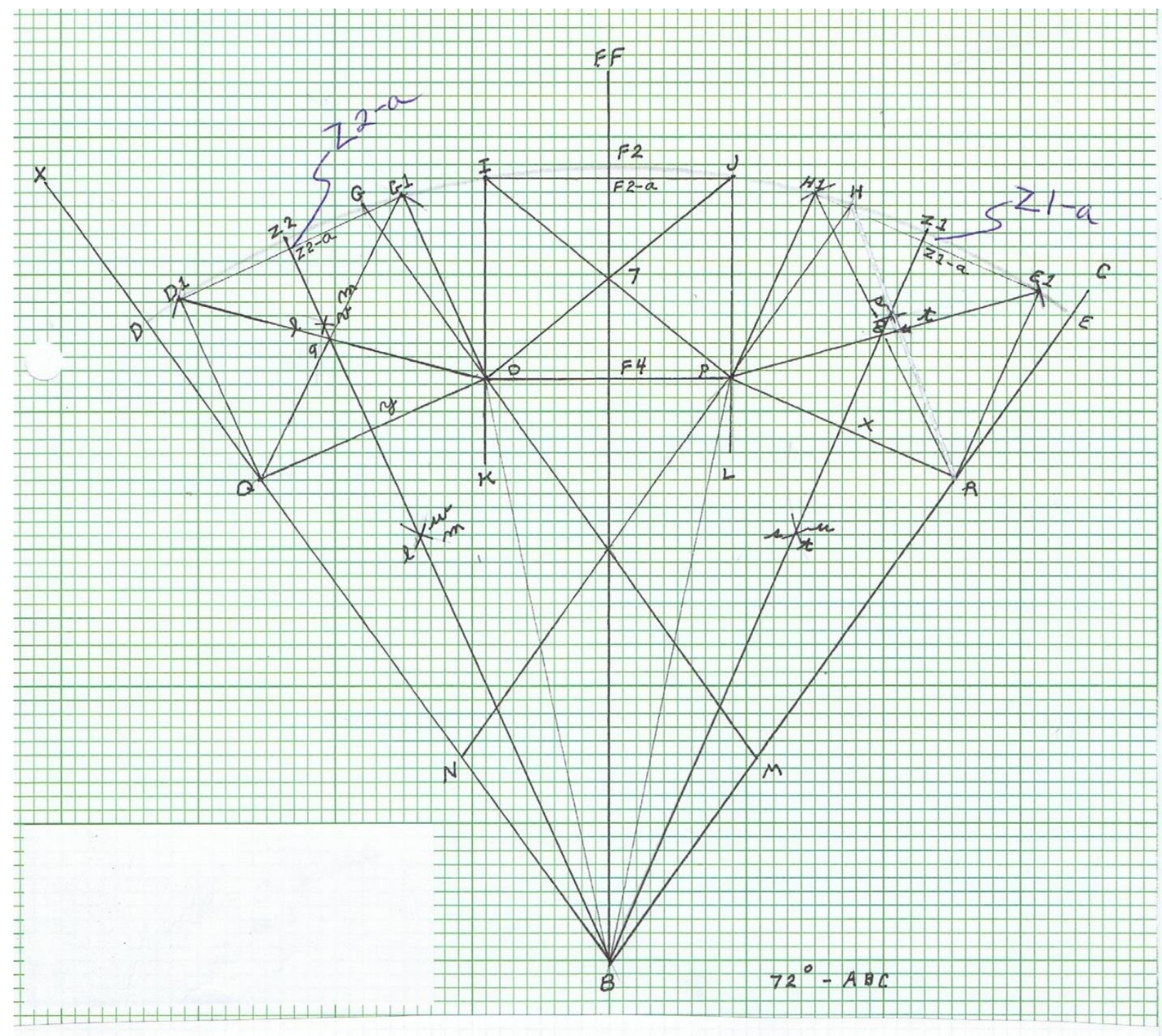

Figure 13: Solving the trisection of a 72 degree angle 


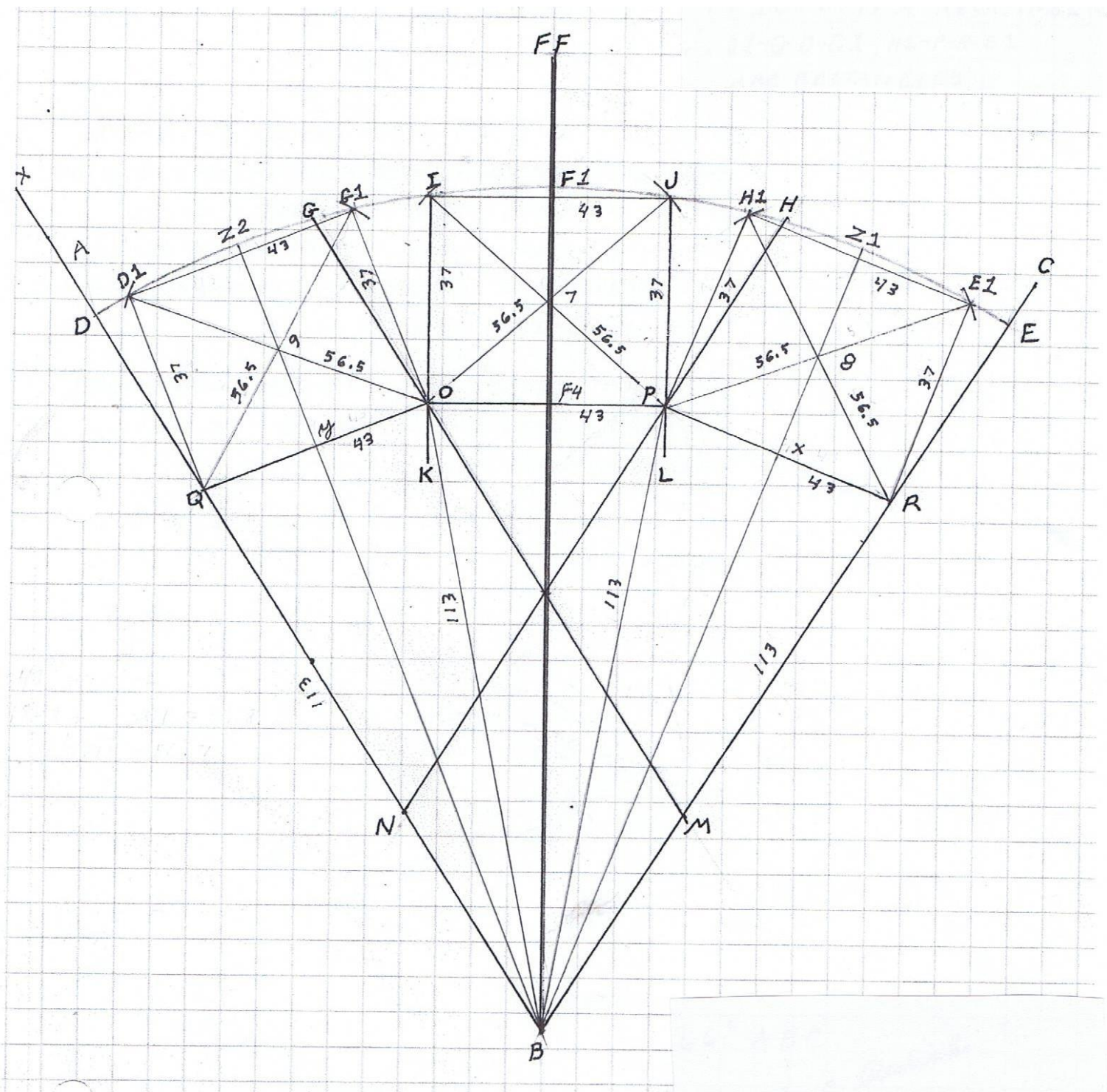

Pythagorean theorem example:

Parallelogram IOPJ

Triangle OPJ

Base $=43$ millimeters

Height $=37$ millimeters
47 squared +37 squared $=3218$

$\sqrt{ } 3218=56.7$

Diagonal OJ

Angle OPJ is 90 degrees.

Therefore, IOPJ is a rectangle.

Therefore, D1-Q-O-G1 \& H1-P-R-E1 are rectangles.

Figure 14: Pythagorean theory example for a 66 degree angle 


\section{CONCLUSIONS}

This paper has presented a multistep classical geometric algorithm for solving the ancient Greek's problem of angle trisection. The offered method shows that, in fact, angle trisection is possible, and has shown that this mathematical problem is geometrically solvable, by providing an elegant solution governed by the classical rules of Euclidean geometry [10]. The involved analogs concern a significant look at elemental geometric quantities (elemental lengths, curves and geometric figures), and their geometrical relationship on a plane. The obtained results justify that the angle trisection impossibility statement, together with the presented nonEuclidean solutions [11], [12], [13], has no geometric rationality. This paper therefore concludes that the angle trisection impossibility statement is not geometrically valid, and that the angle trisection problem is resolvable following the provided method.

\section{Conflict of Interest}

No conflict of interest exists.

\section{ACKNOWLEDGMENTS}

I wish to acknowledge my high school algebra teacher, Miss Sloan (Yakima High School, Yakima, Washington, 1941), for her help in making me understand the basic mathematical concepts. I also appreciate the unstinting support of my blog master, Maitri Sojourner, for making my solution accessible to a global audience, and for final formatting and layout. I wish to thank Claire Thompson for layout and editing of the manuscript. I wish to thank Mr. M. Alex Kimuya for his assistance in re-stating the classic mathematical puzzle and P.L. Wantzel's 'impossibility' claim; and for Mr. Kimuya's explanation that Wantzel's claim is algebraically-based, while my proof is based strictly on Euclidean geometry. I also wish to thank him for his assistance with typing of the manuscript.

\section{REFERENCES}

[1] C. B. Boyer. 1991. A History of Mathematics, 2nd Ed., John Wiley \& Sons, Inc, (1991), p.64.

[2] P.L. Wantzel. 1837. Recherches sur les moyens de reconnaitre si un problème de géométrie peut se résoudre avec la règle et le compass. Journal de Mathematiques pures et appliques, (1837), Vol. 2, pp.366-372.

[3] Kimuya .M. Alex, Josephine Mutembei, The Cube Duplication Solution (A Compass-straightedge (Ruler) Construction), International Journal of Mathematics Trends and Technology (IJMTT) - Volume 50 Number (5 October 2017).

[4] University of Toronto. 1997. The Three Impossible Constructions of Geometry, < mathnet@math.toronto.edu>, 14 Aug, (1997).

[5] Kimuya .M. Alex, Josephine Mutembei, The Angle Trisection Solution (A Compass-Straightedge (Ruler) Construction), Journal of Advances in Mathematics, (2017).

[6] Kimuya, M. Alex. 2017 The Possibility of Angle trisection (A Compass Straightedge Construction), Journal of Mathematics and System Science, Vol.7, (January, 25, 2017), P. 25-42. doi: 10.17265/2159-5291/2017.01.003.

[7] W.S. Anglin. 1994. Mathematics: A Concise History and Philosophy, Springer-Verlag, New York, (1994), pp. 75-80.

[8] H.Dorrie. 1965. 100 Great Problems of Elementary Mathematics, Dover Publications, New York, (1965), P. 173. 
[9] J. Castellanos. 1994-2007. What is Non-Euclidean Geometry? <https://www.cs.unm.edu/ joel/NonEuclid/noneuclidean.html>, (1994)-(2007).

6. Burton, M. David. 1999. A History of Mathematics: An Introduction, 4th Ed., McGraw-Hill, p. 116, (1999).

[10] R. Descartes. 1664. La Géométrie, C. Angot, Paris, (1664) (first published in 1637).

[11] R. Fitzpatrick. 1883-1885. Euclid's Elements of Geometry, from Euclidis Elementa, edidit et Latine interpretatus est I.L. Heiberg, in aedibus, B.G. Teubneri.

[12] H. Florentino Latortue. 2017. The Solution to the Impossible Problem, Library of Congress Control Number: 2017905297, NY, Printed in the United States of America, (2017).

[13] C.A.Hart and D. D. Feldman. 1912, 2013. Plane and Solid Geometry, American Book Company, Chicago, (1912), (2013), p.341-342.

[14] Dudley, U. 1983. What to do when the trisection comes. The Mathematical Intelligencer 5, (1), (1983), p.21.

[15] Dudley, Underwood. 1994. The Trisectors, The Mathematical Association of America.

[16] Kimuya, M. Alex. 2017. Personal communication.

\section{Preparation of Figures}

All figures of the proof itself (Figures 1-10) were originally hand-drawn by the author, using only a straightedge and a compass. Per the rules of the mathematical puzzle, no marks on the straight-edge were used, or were any degree marks or marks of any kind used on the compass to make the drawings of the proof itself (Figures 1-10). The drawings were then scanned to create PDF files for inclusion in the manuscript. The PDF source files for the figures are available upon request. 


\section{SUPPLEMENTARYMATERIALS}

\section{ANNEX 1: The Mistake Made in Proving the Angle Trisection Impossibility Statement (Wantzel, 1837)}

There is much to be found on the internet, in university libraries, and other resources on the angle trisection problem and its solutions. Various authors have taken different approaches to the problem. They have all exhibited degrees of misconception in their attempts to solve the problem. Among the most influential accounts on the angle trisection problem is P.L.Wantzel's proof of the "impossibility" which states "the trisection of an angle corresponds to resolving a certain cubic equation, whose solution cannot be sought geometrically." In his proof, Wantzel used ideas from abstract algebra in an attempt to demonstrate that the problem cannot be solved, as the governing cubic equation cannot be factorized geometrically. This paper is aimed at refuting P.L Wantzel's claim by exposing a purely classical construction of trisecting any angle. As in the case of angle trisection, consider the following relation (sourced from [3], showing that no algebraic proof is correct in justifying a geometric impossibility, and so is the angle trisection proof of impossibility:

Assumed that $\sqrt{2}$ is irrational as it is algebraically believed. Therefore, suppose that $\sqrt{2}$ is rational, then, $\sqrt{2}$ can be written as

$\sqrt{2}=a / b$, where both $a$ and $b$ are whole numbers with no common factor. It follows that;

$2=(a / b)^{\wedge} 2$

Therefore, $2\left(b^{2}\right)=\left(a^{2}\right)$

Clearly, following the algebraic fact that;

a. The product of two odd numbers is odd

b. The product between an odd number and an even number is even

c. The product between two even numbers is even

It can be deduced that if both $a$ and $b$ have no common factor, then the relation $2\left(b^{2}\right)=\left(a^{2}\right)$ poses a contradiction, since the product $2\left(b^{\wedge} 2\right)$ is even, because of the number 2 . This implies that $a$ itself must be even. Therefore, it is not possible to construct the factor $\sqrt{2}$, which is classified as irrational.

\section{ANNEX 2: Geometric construct of $\sqrt{ } 2$}

From Figure (15), it is evident that, the factor $\sqrt{2}$ is geometrically constructible. The fact that the value of $\sqrt{2}$ cannot be algebraically determined, the geometric interpretation of this case is that, the factor $\sqrt{2}$ is a multiplicative factor, which propagate along any such two dimensional figure of the kind shown in Figure (15). Thus in this case, the impossibility proof presented in section 1.4 is false in the sense that, it provides proof to a statement, and not a construction. This is in harmony with Euclid's geometrical proof of the Pythagoras theorem governed by the equation $c^{2}=a^{2}+b^{2}$, that it is geometrically possible to construct the diagonals of a square, and that it is possible to double the content (area) of a square. The algebraic consideration of this case remain an impossibility, which is not geometrically justified. One can therefore make a similar conclusion to the angle trisection impossibility, which is established based on algebra, that it is not geometrically valid to term the angle trisection problem as impossible, without a geometric proof of the claim. Figure (15) show the construction of the factor $\sqrt{2}$. 


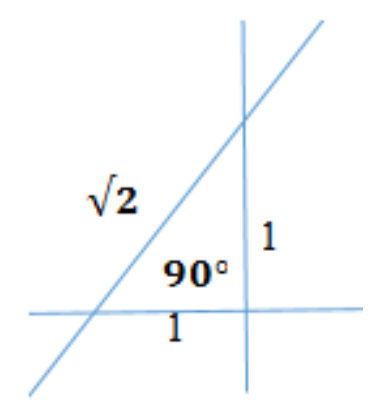

Figure 15: Geometrical construction of the irrational number $\sqrt{2}$ [16]

\section{ANNEX 3: Theoretical Foundation of the Proposed Construction ("Reasons")}

1. Two rays intersect to form an angle. See Figure 1.

2. Segment $\overline{X A}$ used later in proof.

3. An arc may be drawn with a compass set to a known or unknown opening with the sharp point on a given point and the arc length may or may not be given.

4. See Reason 3.

5. See Reason 3.

6. Construction. See Figure 1. See Reason 3. Information statement.

7. See Reason 3.

8. See Reason 3.

9. A collinear line may have 4 points. Reason 19.

10. Construction.

11. Both drawn with same compass opening. Reason 23.

12. Three sides congruent (SSS). Reason 21.

13. CPCTC. Corresponding parts congruent. Reason 22.

14. Angles $D B 30$ and $E B 30$ are adjacent and congruent.

15. Drawn with the same compass opening. Reason 23.

16. An arc has only one chord. Reason 24.

17. Arcs were congruent.

18. Construction. See Figure 1. See NOTE-1. See Reason 3.

19. See Reason 3.

20. Construction.

21. A collinear line may have 3 points. Reason 19.

22. Both drawn with same compass opening. Reason 23.

23. Both drawn with same compass opening. Reason 23.

24. Three sides congruent (SSS). Reason 21

25. CPCTC. Corresponding parts congruent. Reason 22.

26. Angles $E B 31$ and $F B 31$ are adjacent and congruent.

27. Drawn with same compass opening. Reason 23.

28. An arc has only one chord. Reason 24.

29. Arcs were congruent. Information statement.

30. Construction. See Figure 1. See Reason 3.

31. See Reason 3.

32. Construction.

33. A collinear line may have 3 points. Reason 19.

34. Both drawn with same compass opening. Reason 23.

35. Both drawn with same compass opening. Reason 23. 
36. Three sides congruent (SSS). Reason 21.

37. CPCTC. Corresponding parts are congruent. Reason 22. Triangles are congruent.

38. Angles $32 F B$ and $32 D B$ are adjacent and congruent.

39. Drawn with same compass opening. Reason 23.

40. An arc has only one chord. Reason 24.

41. Arcs were congruent.

42. Construction. See Figure 1. See NOTE 1. See Reason 3.

43. See Reason 3.

44. Construction.

45. A collinear line may have three points. Reason 19.

46. Both were drawn with same compass opening. Reason 23.

47. Both were drawn with same compass opening. Reason 23.

48. Three sides congruent (SSS). Reason 21.

49. СРСTC. Reason 21.

50. Angles $35 H B$ and $35 E B$ are adjacent and congruent.

51. Drawn with same compass opening. Reason 23.

52. An arc has only one chord. Reason 24.

53. Arcs were congruent.

54. Construction. See Figure 2. See Reason 3.

55. See Reason 3.

56. Construction.

57. A collinear line may have three points. Reason 19.

58. Both drawn with same compass opening. Reason 23.

59. Three sides congruent (SSS). Reason 21.

60. Drawn with same compass opening.

61. An arc has only one chord. Reason 24.

62. Arcs were congruent.

63. Construction. See Figure 2. See Reason 3.

64. See reason 3 .

65. Construction.

66. A collinear line may have three points. Reason 19.

67. Both sides drawn, same compass opening. Reason 23.

68. (SSS). Reason 21.

69. CPCTC. Reason 22.

70. $\angle 34 G B$ and $\angle 34 F B$ are adjacent and congruent.

71. An arc may have one chord. Reason 24.

72. Both sides drawn with same compass opening. Reason 23.

73. Arcs were congruent.

74. Construction. See Figure 2. See Reason 3.

75. Construction.

76. A collinear line may have three points. Reason 19.

77. Both drawn, same compass opening. Reason 23.

78. Angles $G B I$ and $I B F$ are adjacent and congruent.

79. CPCTC. Reason 22.

80. СРCTC. Reason 22.

81. СРCTC. Reason 22.

82. Construction. Collinear line may have 3 points. See Figure 3. Reason 19.

83. An arc can have only one chord. Reason 24.

84. Construction. Collinear line may have 3 points. See Figure 3. Reason 19.

85. An arc can have only one chord. See Figure 3. Reason 24.

86. Subtraction property of equality. See Figure 3.

87. Addition property of equality. See Figure 4. 
88. An arc can have only one chord. See Figure 4. Reason 24.

89. Congruent arcs have congruent chords. See Figure 4. Reason 25.

90. Construction. See Figure 3.

91. Construction. See Figure 3. See Reason 3.

92. Construction. See Figure 3. See Reason 3.

93. Two points determine a line. Reason 28.

94. See Figure 1. Reason 3.

95. Two points determine line or segment. Reason 27. A collinear line may have 3 points. See Figure 3. Reason 20.

96. Construction. See Figure 3.

97. Two lines parallel, if cut by a transversal such that two corresponding $\angle s$ are congruent. Reason 12 .

98. Construction. See Figure 3. See Reason 3.

99. Construction. See Figure 3.

100. Construction. See Figure 3.

101. Two points determine a line or segment. See Reason 28.

102. Construction. See Reason 3.

103. Construction. See Figure 3.

104. Construction. See Figure 3.

105. Two lines parallel, if cut by a transversal such that two corresponding $\angle s$ are congruent. See Reason 12.

106.Construction. See Figure 4. A collinear line may have 3 points. Reason 19.

107. Point $Z$ is the midpoint of Line IJ.

108. Construction. See Figure 4.

109. Construction. See Reason 3.

110.Construction. See Reason 3.

111.Construction.

112.Construction. See Reason 3.

113. Construction. See Reason 3.

114.Construction. See Reason 3.

115. Construction. See Reason 3.

116. A collinear line may have 4 points. See Reason 19.

117. Two lines parallel, if cut by a transversal such that two corresponding $\angle s$ are congruent. See Reason 12.

118.A collinear line may have 4 points. See Reason 19.

119. Two lines parallel to third line are parallel to each other. See Reason 30.

120.Construction. See Figure 4. Two points determine a line or segment. See Reason 28.

121. Opposite sides are parallel. Mirror image.

122. Opposite sides are congruent.

123.Same compass opening.

124.Same compass opening.

125. By definition.

126. Mirror image.

127. Pair of sides parallel and congruent.

128. Pair of sides parallel and congruent.

129. Two points determine a line. Figure 7. See Note-5. Common hypotenuse, congruent heights. Figure 7.

130.Two points determine a line. Figure 7. See Note-5. Common hypotenuse, congruent heights. Figure 7.

131. Two points determine a line. Figure 7. See Note-5. Common hypotenuse, congruent heights. Figure 8.

132. Two points determine a line. Figure 7. See Note-5. Common hypotenuse, congruent heights. Figure 8.

133. Opposite sides congruent, four right $\angle$. Figure 11.

134. Opposite sides congruent, four right $\angle$. Figure 11.

135. Reason 27.

136. Information statement. 
137. Figure 6. Reason 3.

138. Figure 6. Reason 11

139. Two points determine a line. Reason 28.

140. Four straight sides. Figure 6.

141. Figure 6. Reason 3.

142. Figure 6. Reason 3.

143. Figure 6. Reason 3.

144. Collinear line, have 5 points. Reason 19.

145. Figures 3 and 7 . Reason 3.

146. Figure 6. Reason 13.

147.Figures 3 and 7. Reason 3.

148. Figure 6. Reason 13.

149. Two points determine a line. Reason 28.

150.Two points determine a line. Figure 8. See Reason 28. Common hypotenuse, congruent heights.

151.Two points determine a line. Figure 9. See Reason 28. Common hypotenuse, congruent heights.

152. Two points determine a line. Figure 9. See Reason28. Common hypotenuse, congruent heights.

153. Two points determine a line. Figure 9. See Reason 28. Common hypotenuse, congruent heights.

154.Opposite sides congruent, four right $\angle$. Figure 10.

155. Opposite sides congruent, four right $\angle$. Figure 10.

156. Reason 2.

157.Sum of addition. Figure 11.

158. Information statement.

159. Mirror image. Construction. Figure 11.

160. Rectangles congruent.

161. Same compass opening.

162.SSS.

163. CPCTC.

164. Proof completed.

\section{ANNEX 4: Classical Geometric Reasons Governing the Construction}

1. Two rays intersect to form an angle.

2. (Segment XA used later in proof.)

3. An arc may be drawn with a compass set to a known or unknown opening with the sharp point on a given point and the arc length may or may not be given.

4. Mirror image - Each side of the fiducial line is the reflection of the other side.

5. Corresponding line segments and angles of the mirror image are congruent.

6. A mirror image is of the same size as the original object.

7. Reflexive Property - Any segment or angle is congruent to itself.

8. A quadrilateral has four straight sides.

9. A parallelogram has two pairs of parallel sides, opposite sides are congruent.

10. A parallelogram has four sides, if one pair of opposite sides are congruent, then the other pair of opposite sides are congruent.

11. A rectangle has all the attributes of a parallelogram and at least one angle must be a right angle.

12. If a traverse intersects two parallel lines, alternate interior angles are congruent.

13. Open a compass to the length of a segment, then using that amount of opening, mark off another segment of the same length.

14. A compass can be used to copy an angle.

15. A compass can be used to bisect angles and segments.

16. A compass can be used to construct the perpendicular bisector of a segment.

17. A compass can be used to construct a line perpendicular to a given line through a point on a given line. 
18. A compass can be used to construct a line perpendicular to a given line through a point not on the given line.

19. A collinear line may have 3 or more points.

20. Construction.

21. Three sides congruent (SSS).

22. CPCTC - Corresponding parts of congruent triangles are congruent.

23. Both were drawn with the same compass opening.

24. An arc can have only one chord.

25. Congruent arcs have congruent chords.

26. Subtraction property of equality.

27. Addition property of equality.

28. Two points determine a line or segment.

29. Two lines are parallel if they are cut by a transversal such that two corresponding angles are congruent.

30. Two lines parallel to a third line are parallel to each other.

31. By definition.

32. Reflection line.

33. If a radius is perpendicular to a chord, then it bisects the chord.

34. If a radius bisects a chord, then it is perpendicular to the chord.

35. If two chords of a circle are equidistant from the center of the circle, then they are congruent.

36. If two chords of a circle are congruent, then they are equidistant from the center.

Proofs: Author will comply with the requirement that corrected proofs must be returned to the publisher within two to three days of receipt, and understands that the publisher will do everything possible to ensure prompt publication.

\section{Author's Biography with Photo}

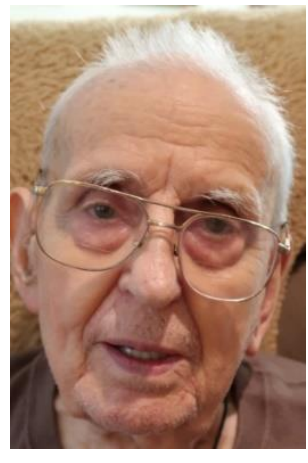

Arthur Clair Rediske is 95 years old. He has worked in various companies including: General Electric, Douglas United Nuclear, Kaiser Engineering and Vitro, Bechtel Corp. He worked as a Field Engineer in a welding lab. He worked as a certified inspector for electrical and instrument installations. Mr. Rediske was a certified inspector for electrical and instrument installations for " $\mathrm{N}$ " nuclear reactor and worked on all phases of instrumentation for " $\mathrm{N}$ " reactor startup. He has worked with expert manual TIG arc welders, and with master welders using automated welding machines. He served as past president of the Richland, WA branch of the ISA, Instrument Society of America. Interestingly, Mr. Rediske invented a method of measuring the number of rotations an arrow makes as it travels to a target. Mr. Rediske has been designing and making sterling silver jewelry since 1940 .

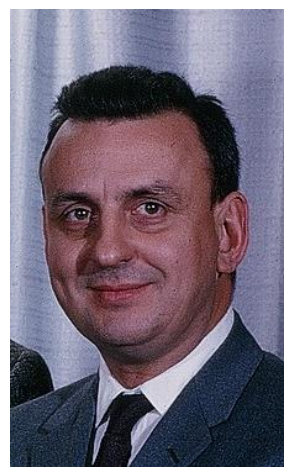

Mr. Rediske, while employed at Bechtel Corporation in their weld lab at Hanford, Washington, USA, wrote the first calibration and maintenance manual for the Astro-arc automatic pipe welding system. This manual, commissioned by the Atomic Energy Commission, did not exist prior to Mr. Rediske's effort. Mr. Rediske also wrote a calibration and maintenance manual for tubing welders used at " $\mathrm{N}$ " nuclear reactor, Hanford, WA. He has published several articles in the Instrument Society of America (ISA) journal, ISA December 1962, Hybrid Instruments, Hanford Library HW-SA-2674 Hanford Library HW-54-2724. He has also published articles in the ISA magazine that are available in the Hanford Library in Richland, Washington. He has also published articles in woodworking and archery magazines. 